九州大学学術情報リポジトリ

Kyushu University Institutional Repository

\title{
Arithmetic of the splitting field of Alexander polynomial
}

Komatsu, Toru

Faculty of Mathematics, Kyushu University

http://hdl. handle. net/2324/3390

出版情報：MHF Preprint Series. 2006-17，2006-04-03. 九州大学大学院数理学研究院 バージョン：

権利関係 : 


\title{
MHF Preprint Series
}

Kyushu University

21st Century COE Program

Development of Dynamic Mathematics with High Functionality

\section{Arithmetic of the splitting field of Alexander polynomial}

\author{
T. Komatsu
}

MHF 2006-17

( Received April 3, 2006 )

Faculty of Mathematics

Kyushu University

Fukuoka, JAPAN 


\title{
Arithmetic of the splitting field of Alexander polynomial
}

\author{
Toru KOMATSU
}

\section{$\S 1$. Introduction}

In this paper we study the arithmetic of the minimal splitting field of the Alexander polynomial of a knot and present two kinds of infinite families of knots, one is a family of knots which satisfying Heilbronn conjecture (Conjecture 1.3) and the other is a family of counterexamples to the conjecture.

For a knot $K$ in $\mathbb{R}^{3}$ let us denote by $\Delta_{K}(t)$ the Alexander polynomial of $K$. In general, $\Delta_{K}(t)$ is defined up to $\pm t^{k}$. In this paper we assume that $\Delta_{K}(t)$ is normalized so that $\Delta_{K}(t)$ is a polynomial in $\mathbb{Z}[t]$ whose order at $t=0$ is equal to 0 . It is a difficult problem to determine the period of a knot $K$ in terms of the Alexander polynomial $\Delta_{K}(t)$. However, some necessary conditions for a knot to have some period are known. For a positive integer $m \in \mathbb{Z}, m \geq 1$ let $\zeta_{m}$ be a primitive $m$-the root of unity in $\overline{\mathbb{Q}}$. Let $F_{f}$ be the minimal splitting field of a polynomial $f \in \mathbb{Q}[t]$ over $\mathbb{Q}$.

Proposition 1.1 (Trotter $[\mathbf{7}]$ ). Let $K$ be a fibered knot with $\operatorname{disc}_{t} \Delta_{K}(t) \neq 0$. If $K$ has period $m$, then $\zeta_{m} \in F_{\Delta_{K}}$.

Let $p$ be a prime number. For a positive integer $\lambda \in \mathbb{Z}, \mathbb{Z} \geq 1$ and a polynomial $\mu \in \mathbb{Z}[t]$ let us denote by $M_{p}(\lambda, \mu)$ the set consisting of polynomials $f \in \mathbb{Z}[t]$ such that $f \equiv \pm t^{k}\left(1+t+\cdots+t^{\lambda-1}\right)^{p-1} \mu^{p}(\bmod p)$ for some integer $k \in \mathbb{Z}$.

Proposition 1.2 (Murasugi [4]). If $K$ is a knot with prime period $p$, then $\Delta_{K}(t) \in M_{p}(\lambda, \mu)$ for a positive integer $\lambda \in \mathbb{Z}, \lambda \geq 1$ and a polynomial $\mu \in \mathbb{Z}[t]$.

Let $H(F)$ be the Hilbert class field of a finite number field $F$.

Conjecture 1.3 (Heilbronn (cf. [5])). For a (fibered) knot $K$, if $\Delta_{K}(t) \in$ $M_{p}(\lambda, 1)$ for a positive integer $\lambda \geq 1$, then $\zeta_{p} \in H\left(F_{\Delta_{K}}\right)$. 
We have already obtained some counterexamples to the Heilbronn's conjecture (e.g., Example 2.3). Morishita gave a question which revises the Heilbronn's conjecture. For a polynomial $f \in \mathbb{Q}[t]$ we say that $f$ satisfies the condition (U) if $\zeta_{p} \in H\left(F_{f}\right)$.

Question 1.4 (Morishita (cf. [5])). What is a condition so that the Alexander polynomial $\Delta_{K}(t)$ with degree $p-1$ of a knot $K$ satisfies the condition (U)?

In this paper we show the following theorem by constructing the explicit Alexander polynomials of knots.

Theorem 1.5 (Corollary 3.4). For every odd prime number $p>3($ resp. $p=3)$ there exist infinitely many knots $K$ whose Alexander polynomials $\Delta_{K}(t)$ are monic (resp. non-monic) of degree $p-1$ and satisfy the two conditions $\Delta_{K}(t) \in M_{p}(2,1)$ and $(\mathrm{U})$.

Theorem 1.6 (Corollary 3.8). For every odd prime number $p>3($ resp. $p=3)$ there exist infinitely many knots $K$ whose Alexander polynomials $\Delta_{K}(t)$ are monic (resp. non-monic) of degree $p-1$ and satisfy the conditions $\Delta_{K}(t) \in M_{p}(2,1)$ but fail the condition (U).

REMARK 1.7. The Alexander polynomial of a fibered knot is monic. For the Alexander polynomial $\Delta_{K}(t)$ of a knot $K$, if $\Delta_{K}(t)$ is monic, then there exists a fibered knot $K^{\prime}$ so that $\Delta_{K^{\prime}}(t)=\Delta_{K}(t)($ Burde $[\mathbf{1}])$.

Acknowledgement. The author is grateful to Professor Masaaki Morishita for introducing the Heilbronn's conjecture. He is grateful to Professor Kunio Murasugi for his many helpful comments. He is supported by the 21st Century COE Program "Development of Dynamic Mathematics with High Functionality".

\section{$\S 2$. Known results}

For a positive integer $n$ let $A_{n}$ be the set of the Alexander polynomials $\Delta_{K}(t)$ of knots $K$ with degree $n$. We define three conditions (A.1) to (A.3) for a polynomial $f(t) \in \mathbb{Z}[t]$ by

(A.1) the degree $n$ of $f(t)$ is even, 
(A.2) $f(t)=t^{n} f\left(t^{-1}\right)$,

(A.3) $f(1)= \pm 1$.

Proposition 2.1 (Seifert $[6]$ ). For a polynomial $f(t) \in \mathbb{Z}[t]$ with degree $n$, it holds that $f(t) \in A_{n}$ if and only if $f(t)$ satisfies all of the three conditions (A.1) to (A.3).

Let $p$ be a prime number. For a positive integer $\lambda \in \mathbb{Z}, \mathbb{Z} \geq 1$ and a polynomial $\mu \in \mathbb{Z}[t]$ let $M_{p}(\lambda, \mu)$ be the set as in Introduction, that is, the set consisting of polynomials $f \in \mathbb{Z}[t]$ such that $f \equiv \pm t^{k}\left(1+t+\cdots+t^{\lambda-1}\right)^{p-1} \mu^{p}(\bmod p)$ for some integer $k \in \mathbb{Z}$. If $f(t) \in A_{n} \cap M_{p}(\lambda, 1)$, then $t^{k}\left(1+t+\cdots+t^{\lambda-1}\right)^{p-1} \equiv f(t)=$ $t^{n} f\left(t^{-1}\right) \equiv t^{n-k}\left(1+t^{-1}+\cdots+t^{-(\lambda-1)}\right)^{p-1}(\bmod p)$. By considering the degrees, one has that $k+(\lambda-1)(p-1)=n-k$, which shows $n=(\lambda-1)(p-1)+2 k$. Note that $k \geq 0$ for $f(t) \in \mathbb{Z}[t]$. Let us calculate the Hilbert class field $H\left(F_{\Delta_{K}}\right)$ of the Alexander polynomials $\Delta_{K}(t)$ of some knots $K$ and determine whether or not the Heilbronn's conjecture holds.

EXAmple 2.2 (cf. [5]). For $\Delta_{K}(t)=4-7 t+4 t^{2} \in A_{2} \cap M_{3}(2,1)$, we have $F_{\Delta_{K}}=\mathbb{Q}(\sqrt{-15})$. One can see that $H\left(F_{\Delta_{K}}\right)=F_{\Delta_{K}}\left(\zeta_{3}\right) \ni \zeta_{3}$. Thus Heilbronn's conjecture is true for the case $\Delta_{K}(t)=4-7 t+4 t^{2}$.

EXAMPLE 2.3 (cf. [5]). When $\Delta_{K}(t)=1-6 t+11 t^{2}-6 t^{3}+t^{4} \in A_{4} \cap M_{5}(2,1)$, we have $F_{\Delta_{K}}=\mathbb{Q}(\sqrt{5})$ since $\Delta_{K}(t)=\left(1-3 t+t^{2}\right)^{2}$. Note that $H(\mathbb{Q}(\sqrt{5}))=\mathbb{Q}(\sqrt{5})$. This means that $\zeta_{5} \notin H\left(F_{\Delta_{K}}\right)$, that is, Heilbronn's conjecture is not true for the case $\Delta_{K}(t)=1-6 t+11 t^{2}-6 t^{3}+t^{4}$.

We define four conditions $(\mathrm{U}),\left(\mathrm{U}_{1}\right),\left(\mathrm{U}_{2}\right)$ and $\left(\mathrm{U}_{3}\right)$ for a finite Galois extension $F$ of $\mathbb{Q}$ such that

(U) $\zeta_{p} \in H(F)$,

$\left(\mathrm{U}_{1}\right) F\left(\zeta_{p}\right) / F$ is unramified,

$\left(\mathrm{U}_{2}\right) F\left(\zeta_{p}\right) / F$ is unramified at all the prime ideals $\mathfrak{p}$ of $F$ above $p$,

$\left(\mathrm{U}_{3}\right)$ for every prime ideal $\mathfrak{p}$ of $F$ above $p$, the ramification index of $\mathfrak{p}$ in the extension $F / \mathbb{Q}$ is a multiple of $p-1$.

The ramification theory of algebraic number theory implies 
Lemma 2.4. Assume that $p$ is odd. If $F$ is totally real, then $F\left(\zeta_{p}\right) / F$ is ramified at all infinite places of $F$, in particular, $\zeta_{p} \notin H(F)$. When $F$ is totally imaginary, the four conditions $(\mathrm{U}),\left(\mathrm{U}_{1}\right),\left(\mathrm{U}_{2}\right)$ and $\left(\mathrm{U}_{3}\right)$ are equivalent to each other.

Morishita and Taguchi showed a sufficient condition to hold (U). For a polynomial $g(t)=\sum_{i=0}^{n} a_{i} t^{i} \in \mathbb{Z}[t]$ we call $g(t)$ a $p$-Eisenstein polynomial if the coefficients $a_{i}$ satisfy that $p \nmid a_{n}, p \mid a_{i}$ for $0 \leq i \leq n-1$ and $p^{2} \nmid a_{0}$. In this paper we do not assume that a $p$-Eisenstein polynomial is monic.

Proposition 2.5 (Morishita-Taguchi (cf. [5])). If $g(t) \in \mathbb{Z}[t]$ is a p-Eisenstein polynomial of degree $p-1$, then $\zeta_{p} \in H\left(F_{g}\right)$.

EXAMPLE 2.6 (cf. [5]). If $g(t)=t^{4}+5 t^{3}-40 t^{2}+70 t-35$, then $g(t+1)=$ $t^{4}+9 t^{3}-19 t^{2}+9 t+1$, which is the Alexander polynomial of some knot. One can see that $F_{g}$ is a $\mathcal{D}_{4}$-extension of $\mathbb{Q}$ where $\mathcal{D}_{4}$ is the dihedral group of degree 4 with order 8 . Note that $F_{g}$ has no subfields which are cyclic quartic fields since $\mathcal{D}_{4}$ has no normal subgroups $N$ such that $\mathcal{D}_{4} / N$ are isomorphic to the cyclic group $\mathcal{C}_{4}$ of order 4 . Thus we have $\zeta_{5} \notin F_{\Delta_{K}}$. Proposition 1.1 implies that $K$ does not have period 5. On the other hand, Proposition 2.5 yields $\zeta_{5} \in H\left(F_{\Delta_{K}}\right)$.

For the use of Proposition 2.5 one needs a criterion whether or not a $p$-Eisenstein polynomial $g(t)$ can become the Alexander polynomial $\Delta_{K}(t)$ of a knot $K$ by some translation $t \mapsto a t+b$ for integers $a$ and $b \in \mathbb{Z}$ with $a \neq 0$, that is, $g(a t+b)=\Delta_{K}(t)$. However, such a criterion is not easy in general. By using a characterization of the Alexander polynomials we present a family of the Alexander polynomials which become $p$-Eisenstein polynomials in the next section.

\section{$\S 3$. Construction of the Alexander polynomials}

For a positive and even integer $n=2 r$ let $B_{n}$ be the set of polynomials $f(t)$ in $\mathbb{Z}[t]$ such that $f(t)=t^{n} f\left(t^{-1}\right)$. Note that $A_{n}=\left\{f(t) \in B_{n} \mid \operatorname{deg}_{t} f(t)=n, f(1)=\right.$ $\pm 1\}$.

Lemma 3.1 (cf. Crowell-Fox $[\mathbf{3}]$ ). The set $B_{n}$ is a $\mathbb{Z}$-module with free basis $\left\{\left(t^{2}-2 t+1\right)^{i} t^{r-i} \mid 0 \leq i \leq r\right\}$, that is, $B_{n}=\left\{\sum_{0 \leq i \leq r} b_{i}\left(t^{2}-2 t+1\right)^{i} t^{r-i} \mid b_{i} \in \mathbb{Z}\right\}$. 
Let $p$ be a prime number and $\lambda \geq 1$ a positive integer such that $n \geq(\lambda-1)(p-1)$. We define a polynomial $\alpha_{\lambda}(t)=\alpha_{n, p, \lambda}(t)$ by

$$
\alpha_{\lambda}(t)=t^{k}\left(1+t+\cdots+t^{\lambda-1}\right)^{p-1}-\left(\lambda^{p-1}-1\right) t^{r}
$$

where $k=r-(\lambda-1)(p-1) / 2$. For integers $c_{i} \in \mathbb{Z}$ with $1 \leq i \leq r$ let us denote $\alpha_{\lambda}(t)+p \sum_{1 \leq i \leq r} c_{i}\left(t^{2}-2 t+1\right)^{i} t^{r-i}$ by $f_{\lambda}(\mathfrak{c}, t)$ where $\mathfrak{c}=\left(c_{1}, c_{2}, \ldots, c_{r}\right)$. We put $S_{r}(\mathbb{Z})=\mathbb{Z}^{r}$ and $S_{r}^{\times}(\mathbb{Z})=\left\{\mathfrak{c}=\left(c_{1}, c_{2}, \ldots, c_{r}\right) \in S_{r}(\mathbb{Z}) \mid c_{r} \neq 0\right\}$.

Lemma 3.2. The intersection set $A_{n} \cap M_{p}(\lambda, 1)$ consists of the polynomials $\pm f_{\lambda}(\mathfrak{c}, t), \mathfrak{c}=\left(c_{1}, c_{2}, \ldots, c_{r}\right)$ where $\mathfrak{c} \in S_{r}(Z)$ if $n=(\lambda-1)(p-1)$ and $\mathfrak{c} \in S_{r}^{\times}(Z)$ otherwise.

Proof. Let $\mathfrak{c}$ be an element in $S_{r}(\mathbb{Z})$ if $n=(\lambda-1)(p-1)$ and in $S_{r}^{\times}(\mathbb{Z})$ otherwise. It follows from the definition that $\operatorname{deg}_{t} f_{\lambda}(\mathfrak{c}, t) \leq n$. If $n=(\lambda-1)(p-1)$, then the coefficient of $t^{p-1}$ in $f_{\lambda}(\mathfrak{c}, t)$ is equal to $1+p c_{r}$. Since $c_{r} \in \mathbb{Z}$, one has that $1+p c_{r} \neq 0$ and $\operatorname{deg}_{t} f_{\lambda}(\mathfrak{c}, t)=n$. When $n>(\lambda-1)(p-1)$, the coefficient of $t^{p-1}$ in $f_{\lambda}(\mathfrak{c}, t)$ is equal to $p c_{r}$. Thus it holds that $\operatorname{deg}_{t} f_{\lambda}(\mathfrak{c}, t)=n$ for $c_{r} \neq 0$. It is easy to see that $\pm f_{\lambda}(\mathfrak{c}, t)$ satisfies the conditions (A.2) and (A.3). Hence the polynomial $\pm f_{\lambda}(\mathfrak{c}, t)$ belongs to $A_{n} \cap M_{p}(\lambda, 1)$. Let $f(t)$ be a polynomial in $A_{n} \cap M_{p}(\lambda, 1)$. For a non-negative integer $k \in \mathbb{Z}$ with $k=r-(\lambda-1)(p-1) / 2$, one has $\pm t^{k}(1+t+\cdots+$ $\left.t^{\lambda-1}\right)^{p-1} \in B_{n}$. Since $B_{n}$ is a $\mathbb{Z}$-molude, we have $f(t) \mp t^{k}\left(1+t+\cdots+t^{\lambda-1}\right)^{p-1} \in B_{n}$ and $\left(f(t) \mp t^{k}\left(1+t+\cdots+t^{\lambda-1}\right)^{p-1}\right) / p \in B_{n}$ if $f(1)= \pm 1$, respectively. Lemma 3.1 implies that $f(t)= \pm t^{k}\left(1+t+\cdots+t^{\lambda-1}\right)^{p-1}+p \sum_{0 \leq i \leq r} c_{i}\left(t^{2}-2 t+1\right)^{i} t^{r-i}$ where $c_{i} \in \mathbb{Z}$ for $0 \leq i \leq r$. It follows from $f(1)= \pm 1$ that $\pm \lambda^{p-1}+p c_{0}= \pm 1$ and $c_{0}=\mp\left(\lambda^{p-1}-1\right) / p \in \mathbb{Z}$. Hence $f(t)$ is of the form $\pm f_{\lambda}(\mathfrak{c}, t)$.

For considering Question 1.4 we have $n=p-1 \geq 2$, which means that $\lambda=1$ or 2. Here one has that $\alpha_{1}(t)=t^{(p-1) / 2}$ and $\alpha_{2}(t)=(1+t)^{p-1}-\left(2^{p-1}-1\right) t^{(p-1) / 2}$. Let us define a number $\varepsilon_{p} \in \mathbb{Q}$ by $\varepsilon_{p}=-\left(2^{p-1}-1\right) /(4 p)$. Note that $v_{p}\left(\varepsilon_{p}\right) \geq 0$.

Theorem 3.3. Let $h(X)=\sum_{j=0}^{(p-3) / 2} s_{j} X^{j} \in \mathbb{Z}[X]$ be a polynomial of degree less than $(p-1) / 2$ such that $s_{0} \not \equiv \varepsilon_{p}(\bmod p)$ and $s_{0}>\varepsilon_{p}$. Then

$$
f(t)=\alpha_{2}(t)+p t^{(p-1) / 2}\left(t+t^{-1}-2\right) h\left(t+t^{-1}+2\right)
$$


is a polynomial in $A_{p-1} \cap M_{p}(2,1)$ satisfying $\zeta_{p} \in H\left(F_{f}\right)$. The polynomial $f(t)$ is monic if and only if $s_{(p-3) / 2}=0$.

Proof. It is easy to check that $f(t) \in A_{p-1} \cap M_{p}(2,1)$. In fact, it holds that $f(t)=f_{2}(\mathfrak{c}, t)$ for an element $\mathfrak{c}=\left(c_{1}, c_{2}, \ldots, c_{(p-1) / 2}\right) \in S_{(p-1) / 2}(\mathbb{Z})$ satisfying $\sum_{i=1}^{(p-1) / 2} c_{i}\left(t+t^{-1}-2\right)^{i-1}=\sum_{j=0}^{(p-3) / 2} s_{j}\left(t+t^{-1}+2\right)^{j}$. One has that $f(t-1) \equiv$ $t^{p-1}(\bmod p)$ and $f(-1)=(-1)^{(p+1) / 2}\left(2^{p-1}-1+4 p s_{0}\right)$. The condition $s_{0} \not \equiv \varepsilon_{p}$ $(\bmod p)$ is equivalent to $v_{p}(f(-1))=1$. Thus $f(t-1)$ is a $p$-Eisenstein polynomial of degree $p-1$. Now put $X=t+t^{-1}+2$. Then we have $f(t) / t^{(p-1) / 2}=$ $X^{(p-1) / 2}-\left(2^{p-1}-1\right)+p(X-4) h(X)$, which is denoted by $\tilde{f}(X)$. Here it is seen that $\widetilde{f}(0)=-\left(2^{p-1}-1\right)-4 p s_{0}$ and $\widetilde{f}(4)=1$. It follows from $s_{0}>\varepsilon_{p}$ that $\widetilde{f}(0)<0$. This shows that $\tilde{f}(X)=0$ has a real solution $x$ with $0<x<4$. For a complex number $z \in \mathbb{C}$ with $z+z^{-1} \in \mathbb{R}$, the condition $0<z+z^{-1}+2<4$ holds if and only if $z$ is not real. This means that $f(t)$ has a non-real zero in $\mathbb{C}$. Thus $F_{f}$ is not totally real but totally imaginary. Hence Lemma 2.4 verifies that $\zeta_{p} \in H\left(F_{f}\right)$.

Corollary 3.4 (Theorem 1.5). For every odd prime number $p>3($ resp. $p=3)$ there exist infinitely many knots $K$ whose Alexander polynomials $\Delta_{K}(t)$ are monic (resp. non-monic) and satisfy the two conditions $\Delta_{K}(t) \in A_{p-1} \cap M_{p}(2,1)$ and $(\mathrm{U})$. Proof. For example, one may take an arbitrary integer $s \in \mathbb{Z}$ satisfying $s \not \equiv \varepsilon_{p}$ $(\bmod p)$ and $s>\varepsilon_{p}$ for the polynomial $h(X)$ in Theorem 3.3.

EXAmple 3.5 (Examples 2.2 and 2.6). For the case that $p=3$ and $h(X)=1$ in Theorem 3.3, one has $f(X)=4 t^{2}-7 t+4$. Since $\varepsilon_{3}=-1 / 4 \equiv 2(\bmod 3)$, we have $\zeta_{3} \in H\left(F_{f}\right)$. If $p=5$ and $h(X)=1$, then $f(t)=t^{4}+9 t^{3}-19 t^{2}+9 t+1$ and $\zeta_{5} \in H\left(F_{f}\right)$ for $\varepsilon_{5}=-3 / 4 \equiv 3(\bmod 5)$.

Theorem 3.6. Assume $p \geq 5$. Let $h(X)=\sum_{i=0}^{(p-3) / 2} s_{i} X^{i} \in \mathbb{Z}[X]$ be a polynomial of degree less than $(p-1) / 2$. If $s_{0} \equiv \varepsilon_{p}(\bmod p)$ and $s_{1} \not \equiv \varepsilon_{p} / 4(\bmod p)$, then $f(t)=\alpha_{2}(t)+p t^{(p-1) / 2}\left(t+t^{-1}-2\right) h\left(t+t^{-1}+2\right)$ is a polynomial in $A_{p-1} \cap M_{p}(2,1)$ such that $\zeta_{p} \notin H\left(F_{f}\right)$. The polynomial $f(t)$ is monic if and only if $s_{(p-3) / 2}=0$.

Proof. In the same way as in the proof of Theorem 3.3 one sees $f(t) \in A_{p-1} \cap$ $M_{p}(2,1)$. For $0 \leq j \leq p-1$ let $\gamma_{j} \in \mathbb{Z}$ be integers such that $f(t-1)=\sum_{j=0}^{p-1} \gamma_{j} t^{j}$. 
It is calculated that

$$
\begin{aligned}
& \gamma_{0}=(-1)^{(p+1) / 2} 4 p\left(s_{0}-\varepsilon_{p}\right) \\
& \gamma_{1}=(-1)^{(p-1) / 2} 2 p(p-1)\left(s_{0}-\varepsilon_{p}\right) \\
& \gamma_{2}=(-1)^{(p-1) / 2} 4 p\left(s_{1}-\frac{p^{2}-4 p+5}{8} s_{0}-\frac{\left(2^{p-1}-1\right)(p-1)(p-3)}{32 p}\right) .
\end{aligned}
$$

By the condition $s_{0} \equiv \varepsilon_{p}(\bmod p)$ we have that $v_{p}\left(\gamma_{0}\right)=v_{p}\left(\gamma_{1}\right) \geq 2$ and $\gamma_{2} \equiv$ $(-1)^{(p-1) / 2} 4 p\left(s_{1}-\varepsilon_{p} / 4\right)\left(\bmod p^{2}\right)$. If $s_{1} \not \equiv \varepsilon_{p} / 4(\bmod p)$, then $v_{p}\left(\gamma_{2}\right)=1$. Since $f(t-1) \equiv t^{p-1}(\bmod p)$, it holds that $v_{p}\left(\gamma_{j}\right) \geq 1$ for $3 \leq j \leq p-2$. Using the Newton polygon method (cf. [2]), one can show that the ramification index of every prime ideal of $F_{f}$ above $p$ is equal to $p-3$. Hence we have $\zeta_{p} \notin H\left(F_{f}\right)$ for Lemma 2.4 .

Lemma 3.7. Let us put $f(t)=(3 s+1) t^{2}-(6 s+1) t+3 s+1$ for an integer $s \in \mathbb{Z}$. Then $f(t)$ is a polynomial in $A_{2} \cap M_{3}(2,1)$. The condition $\zeta_{3} \in H\left(F_{f}\right)$ holds if and only if $v_{3}(s+1 / 4)$ is even and $s$ is greater than $-1 / 4$.

Proof. The discriminant of the polynomial $f(t)$ is equal to $-12(s+1 / 4)$, that is, $F_{f}=\mathbb{Q}(\sqrt{-3(s+1 / 4)})$. The ramification index of 3 in $F_{f} / \mathbb{Q}$ is divisible by 2 if and only if $v_{3}(s+1 / 4)$ is even. The field $F_{f}$ is totally imaginary if and only if $s>-1 / 4$. Hence the assertion holds.

Theorem 3.6 and Lemma 3.7 imply

Corollary 3.8 (Theorem 1.6). For every odd prime number $p>3($ resp. $p=3)$ there exist infinitely many knots $K$ whose Alexander polynomials $\Delta_{K}(t)$ are monic (resp. non-monic) and satisfy the conditions $\Delta_{K}(t) \in A_{p-1} \cap M_{p}(2,1)$ but fail the condition (U).

Proof. When $p \geq 5$, for the polynomial $h(X)$ in Theorem 3.6 one may take a polynomial $s_{1} t+s_{0}$ where $s_{1}$ and $s_{0} \in \mathbb{Z}$ are integers such that $s_{0} \equiv \varepsilon_{p}(\bmod p)$ and $s_{1} \not \equiv \varepsilon_{p} / 4(\bmod p)$. In fact, $\varepsilon_{5}=-3 / 4 \not \equiv 0(\bmod 5)$, which means that we can have $s_{(p-3) / 2}=s_{1}=0$ for the case $p=5$. For $p=3$ let us set $s=-\left(3 u^{2}+3 u+1\right)$ in Lemma 3.7 where $u \in \mathbb{Z}$ is an integer. Then one has $f(t)=-((3 u+1) t-(3 u+$ $2))((3 u+2) t-(3 u+1))$, which implies that $F_{f}=\mathbb{Q}$. 
EXAMPLE 3.9 (Example 2.3). When $p=5$ and $h(X)=-2$, one has $f(t)=$ $t^{4}-6 t^{3}+11 t^{2}-6 t+1$. Since $\varepsilon_{5}=-3 / 4 \equiv 3(\bmod 5)$, the polynomial $h(X)=$ $s_{1} X+s_{0}=-2$ satisfies that $s_{0} \equiv \varepsilon_{5}(\bmod 5)$ and $s_{1} \not \equiv \varepsilon_{5} / 4 \equiv 2(\bmod 5)$. Theorem 3.6 shows that $\zeta_{5} \notin H\left(F_{\Delta_{K}}\right)$.

EXAMPLE 3.10. For an integer $s \in \mathbb{Z}$ let us put $f_{s}(t)=t^{4}+(5 s+4) t^{3}-(10 s+$ $9) t^{2}+(5 s+4) t+1$, which is obtained as $p=5$ and $h(X)=s$ in Theorems 3.3 and 3.6. It is calculated that the discriminant $\delta(s)$ of the polynomial $f_{s}(t)$ is equal to $-5^{5}(s+2)^{2}(5 s+6)^{2}(4 s+3)$. Here $f_{-3}(t)$ (resp. $\left.f_{-1}(t)\right)$ have four real (resp. four non-real) zeros. It holds that $f_{-2}(t)=(t-(3+\sqrt{5}) / 2)^{2}(t-(3-\sqrt{5}) / 2)^{2}$. The polynomial $f_{0}(t)$ has two real and two non-real zeros. Thus $F_{f_{s}}$ is totally real (resp. totally imaginary) provided $s=-3,-2$ (resp. $s=-1,0)$. Note that there exist no zeros of $\delta(s)$ in the areas $s \leq-3$ or $s \geq 0$. Hence $F_{f_{s}}$ is totally real (resp. totally imaginary) when $s \leq-2$ (resp. $s \geq-1$ ). Theorems 3.3 and 3.6 with the argument above imply that Heilbronn's conjecture is true for $f_{s}(t)$ if and only if $s \geq-1$ and $s \not \equiv 3(\bmod 5)$.

\section{References}

[1] G. Burde, Alexanderpolynome Neuwirthscher Knoten, Topology 5 (1966), 321-330.

[2] H. Cohen, A course in computational algebraic number theory, Grad. Texts in Math. 138, 1993.

[3] R.H. Crowell, R.H. Fox, Introduction to knot theory, Grad. Texts in Math. 57, 1977.

[4] K. Murasugi, On periodic knots, Comment. Math. Helv. 46 (1971), 162-174.

[5] K. Murasugi, Classical knot invariants and elementary number theory, preprint.

[6] H. Seifert, Über das Geschlecht von Knoten, Math. Ann. 110 (1935), no. 1, 571-592

[7] H.F. Trotter, Periodic automorphisms of groups and knots, Duke Math. J. 28 (1961), 553-557.

(Toru KOMATSU) Faculty of Mathematics, Kyushu University, 6-10-1 Hakozaki Higashiku, FuKuOKA, 812-8581 JAPAN

E-mail address: trkomatu@math.kyushu-u.ac.jp 


\section{List of MHF Preprint Series, Kyushu University 21st Century COE Program Development of Dynamic Mathematics with High Functionality}

\section{MHF2003-1 Mitsuhiro T. NAKAO, Kouji HASHIMOTO \& Yoshitaka WATANABE}

A numerical method to verify the invertibility of linear elliptic operators with applications to nonlinear problems

MHF2003-2 Masahisa TABATA \& Daisuke TAGAMI

Error estimates of finite element methods for nonstationary thermal convection problems with temperature-dependent coefficients

MHF2003-3 Tomohiro ANDO, Sadanori KONISHI \& Seiya IMOTO

Adaptive learning machines for nonlinear classification and Bayesian information criteria

MHF2003-4 Kazuhiro YOKOYAMA

On systems of algebraic equations with parametric exponents

MHF2003-5 Masao ISHIKAWA \& Masato WAKAYAMA

Applications of Minor Summation Formulas III, Plücker relations, Lattice paths and Pfaffian identities

MHF2003-6 Atsushi SUZUKI \& Masahisa TABATA

Finite element matrices in congruent subdomains and their effective use for large-scale computations

MHF2003-7 Setsuo TANIGUCHI

Stochastic oscillatory integrals - asymptotic and exact expressions for quadratic phase functions -

MHF2003-8 Shoki MIYAMOTO \& Atsushi YOSHIKAWA

Computable sequences in the Sobolev spaces

MHF2003-9 Toru FUJII \& Takashi YANAGAWA

Wavelet based estimate for non-linear and non-stationary auto-regressive model

MHF2003-10 Atsushi YOSHIKAWA

Maple and wave-front tracking - an experiment

MHF2003-11 Masanobu KANEKO

On the local factor of the zeta function of quadratic orders

MHF2003-12 Hidefumi KAWASAKI

Conjugate-set game for a nonlinear programming problem 
MHF2004-1 Koji YONEMOTO \& Takashi YANAGAWA

Estimating the Lyapunov exponent from chaotic time series with dynamic noise

MHF2004-2 Rui YAMAGUCHI, Eiko TSUCHIYA \& Tomoyuki HIGUCHI

State space modeling approach to decompose daily sales of a restaurant into time-dependent multi-factors

MHF2004-3 Kenji KAJIWARA, Tetsu MASUDA, Masatoshi NOUMI, Yasuhiro OHTA \& Yasuhiko YAMADA

Cubic pencils and Painlevé Hamiltonians

MHF2004-4 Atsushi KAWAGUCHI, Koji YONEMOTO \& Takashi YANAGAWA

Estimating the correlation dimension from a chaotic system with dynamic noise

MHF2004-5 Atsushi KAWAGUCHI, Kentarou KITAMURA, Koji YONEMOTO, Takashi YANAGAWA \& Kiyofumi YUMOTO

Detection of auroral breakups using the correlation dimension

MHF2004-6 Ryo IKOTA, Masayasu MIMURA \& Tatsuyuki NAKAKI

A methodology for numerical simulations to a singular limit

MHF2004-7 Ryo IKOTA \& Eiji YANAGIDA

Stability of stationary interfaces of binary-tree type

MHF2004-8 Yuko ARAKI, Sadanori KONISHI \& Seiya IMOTO

Functional discriminant analysis for gene expression data via radial basis expansion

MHF2004-9 Kenji KAJIWARA, Tetsu MASUDA, Masatoshi NOUMI, Yasuhiro OHTA \& Yasuhiko YAMADA

Hypergeometric solutions to the $q$ - Painlevé equations

MHF2004-10 Raimundas VIDŪNAS

Expressions for values of the gamma function

MHF2004-11 Raimundas VIDŪNAS

Transformations of Gauss hypergeometric functions

MHF2004-12 Koji NAKAGAWA \& Masakazu SUZUKI

Mathematical knowledge browser

MHF2004-13 Ken-ichi MARUNO, Wen-Xiu MA \& Masayuki OIKAWA

Generalized Casorati determinant and Positon-Negaton-Type solutions of the Toda lattice equation

MHF2004-14 Nalini JOSHI, Kenji KAJIWARA \& Marta MAZZOCCO

Generating function associated with the determinant formula for the solutions of the Painlevé II equation 
MHF2004-15 Kouji HASHIMOTO, Ryohei ABE, Mitsuhiro T. NAKAO \& Yoshitaka WATANABE

Numerical verification methods of solutions for nonlinear singularly perturbed problem

MHF2004-16 Ken-ichi MARUNO \& Gino BIONDINI

Resonance and web structure in discrete soliton systems: the two-dimensional Toda lattice and its fully discrete and ultra-discrete versions

MHF2004-17 Ryuei NISHII \& Shinto EGUCHI

Supervised image classification in Markov random field models with Jeffreys divergence

MHF2004-18 Kouji HASHIMOTO, Kenta KOBAYASHI \& Mitsuhiro T. NAKAO

Numerical verification methods of solutions for the free boundary problem

MHF2004-19 Hiroki MASUDA

Ergodicity and exponential $\beta$-mixing bounds for a strong solution of Lévydriven stochastic differential equations

MHF2004-20 Setsuo TANIGUCHI

The Brownian sheet and the reflectionless potentials

MHF2004-21 Ryuei NISHII \& Shinto EGUCHI

Supervised image classification based on AdaBoost with contextual weak classifiers

MHF2004-22 Hideki KOSAKI

On intersections of domains of unbounded positive operators

MHF2004-23 Masahisa TABATA \& Shoichi FUJIMA

Robustness of a characteristic finite element scheme of second order in time increment

MHF2004-24 Ken-ichi MARUNO, Adrian ANKIEWICZ \& Nail AKHMEDIEV

Dissipative solitons of the discrete complex cubic-quintic Ginzburg-Landau equation

MHF2004-25 Raimundas VIDŪNAS

Degenerate Gauss hypergeometric functions

MHF2004-26 Ryo IKOTA

The boundedness of propagation speeds of disturbances for reaction-diffusion systems

MHF2004-27 Ryusuke KON

Convex dominates concave: an exclusion principle in discrete-time Kolmogorov systems 
MHF2004-28 Ryusuke KON

Multiple attractors in host-parasitoid interactions: coexistence and extinction

MHF2004-29 Kentaro IHARA, Masanobu KANEKO \& Don ZAGIER

Derivation and double shuffle relations for multiple zeta values

MHF2004-30 Shuichi INOKUCHI \& Yoshihiro MIZOGUCHI

Generalized partitioned quantum cellular automata and quantization of classical CA

MHF2005-1 Hideki KOSAKI

Matrix trace inequalities related to uncertainty principle

MHF2005-2 Masahisa TABATA

Discrepancy between theory and real computation on the stability of some finite element schemes

MHF2005-3 Yuko ARAKI \& Sadanori KONISHI

Functional regression modeling via regularized basis expansions and model selection

MHF2005-4 Yuko ARAKI \& Sadanori KONISHI

Functional discriminant analysis via regularized basis expansions

MHF2005-5 Kenji KAJIWARA, Tetsu MASUDA, Masatoshi NOUMI, Yasuhiro OHTA \& Yasuhiko YAMADA

Point configurations, Cremona transformations and the elliptic difference Painlevé equations

MHF2005-6 Kenji KAJIWARA, Tetsu MASUDA, Masatoshi NOUMI, Yasuhiro OHTA \& Yasuhiko YAMADA

Construction of hypergeometric solutions to the $q$ - Painlevé equations

MHF2005-7 Hiroki MASUDA

Simple estimators for non-linear Markovian trend from sampled data:

I. ergodic cases

MHF2005-8 Hiroki MASUDA \& Nakahiro YOSHIDA

Edgeworth expansion for a class of Ornstein-Uhlenbeck-based models

MHF2005-9 Masayuki UCHIDA

Approximate martingale estimating functions under small perturbations of dynamical systems

MHF2005-10 Ryo MATSUZAKI \& Masayuki UCHIDA

One-step estimators for diffusion processes with small dispersion parameters from discrete observations

MHF2005-11 Junichi MATSUKUBO, Ryo MATSUZAKI \& Masayuki UCHIDA

Estimation for a discretely observed small diffusion process with a linear drift 
MHF2005-12 Masayuki UCHIDA \& Nakahiro YOSHIDA

AIC for ergodic diffusion processes from discrete observations

MHF2005-13 Hiromichi GOTO \& Kenji KAJIWARA

Generating function related to the Okamoto polynomials for the Painlevé IV equation

MHF2005-14 Masato KIMURA \& Shin-ichi NAGATA

Precise asymptotic behaviour of the first eigenvalue of Sturm-Liouville problems with large drift

MHF2005-15 Daisuke TAGAMI \& Masahisa TABATA

Numerical computations of a melting glass convection in the furnace

MHF2005-16 Raimundas VIDŪNAS

Normalized Leonard pairs and Askey-Wilson relations

MHF2005-17 Raimundas VIDŪNAS

Askey-Wilson relations and Leonard pairs

MHF2005-18 Kenji KAJIWARA \& Atsushi MUKAIHIRA

Soliton solutions for the non-autonomous discrete-time Toda lattice equation

MHF2005-19 Yuu HARIYA

Construction of Gibbs measures for 1-dimensional continuum fields

MHF2005-20 Yuu HARIYA

Integration by parts formulae for the Wiener measure restricted to subsets in $\mathbb{R}^{d}$

MHF2005-21 Yuu HARIYA

A time-change approach to Kotani's extension of Yor's formula

MHF2005-22 Tadahisa FUNAKI, Yuu HARIYA \& Mark YOR

Wiener integrals for centered powers of Bessel processes, I

MHF2005-23 Masahisa TABATA \& Satoshi KAIZU

Finite element schemes for two-fluids flow problems

MHF2005-24 Ken-ichi MARUNO \& Yasuhiro OHTA

Determinant form of dark soliton solutions of the discrete nonlinear Schrödinger equation

MHF2005-25 Alexander V. KITAEV \& Raimundas VIDŪNAS

Quadratic transformations of the sixth Painlevé equation

MHF2005-26 Toru FUJII \& Sadanori KONISHI

Nonlinear regression modeling via regularized wavelets and smoothing parameter selection 
MHF2005-27 Shuichi INOKUCHI, Kazumasa HONDA, Hyen Yeal LEE, Tatsuro SATO, Yoshihiro MIZOGUCHI \& Yasuo KAWAHARA

On reversible cellular automata with finite cell array

MHF2005-28 Toru KOMATSU

Cyclic cubic field with explicit Artin symbols

MHF2005-29 Mitsuhiro T. NAKAO, Kouji HASHIMOTO \& Kaori NAGATOU

A computational approach to constructive a priori and a posteriori error

estimates for finite element approximations of bi-harmonic problems

MHF2005-30 Kaori NAGATOU, Kouji HASHIMOTO \& Mitsuhiro T. NAKAO

Numerical verification of stationary solutions for Navier-Stokes problems

MHF2005-31 Hidefumi KAWASAKI

A duality theorem for a three-phase partition problem

MHF2005-32 Hidefumi KAWASAKI

A duality theorem based on triangles separating three convex sets

MHF2005-33 Takeaki FUCHIKAMI \& Hidefumi KAWASAKI

An explicit formula of the Shapley value for a cooperative game induced from the conjugate point

MHF2005-34 Hideki MURAKAWA

A regularization of a reaction-diffusion system approximation to the two-phase

Stefan problem

MHF2006-1 Masahisa TABATA

Numerical simulation of Rayleigh-Taylor problems by an energy-stable finite element scheme

MHF2006-2 Ken-ichi MARUNO \& G R W QUISPEL

Construction of integrals of higher-order mappings

MHF2006-3 Setsuo TANIGUCHI

On the Jacobi field approach to stochastic oscillatory integrals with quadratic phase function

MHF2006-4 Kouji HASHIMOTO, Kaori NAGATOU \& Mitsuhiro T. NAKAO

A computational approach to constructive a priori error estimate for finite element approximations of bi-harmonic problems in nonconvex polygonal domains

MHF2006-5 Hidefumi KAWASAKI

A duality theory based on triangular cylinders separating three convex sets in $R^{n}$

MHF2006-6 Raimundas VIDŪNAS

Uniform convergence of hypergeometric series 
MHF2006-7 Yuji KODAMA \& Ken-ichi MARUNO

N-Soliton solutions to the DKP equation and Weyl group actions

MHF2006-8 Toru KOMATSU

Potentially generic polynomial

MHF2006-9 Toru KOMATSU

Generic sextic polynomial related to the subfield problem of a cubic polynomial

MHF2006-10 Shu TEZUKA \& Anargyros PAPAGEORGIOU

Exact cubature for a class of functions of maximum effective dimension

MHF2006-11 Shu TEZUKA

On high-discrepancy sequences

MHF2006-12 Raimundas VIDŪNAS

Detecting persistent regimes in the North Atlantic Oscillation time series

MHF2006-13 Toru KOMATSU

Tamely Eisenstein field with prime power discriminant

MHF2006-14 Nalini JOSHI, Kenji KAJIWARA \& Marta MAZZOCCO

Generating function associated with the Hankel determinant formula for the solutions of the Painlevé IV equation

MHF2006-15 Raimundas VIDŪNAS

Darboux evaluations of algebraic Gauss hypergeometric functions

MHF2006-16 Masato KIMURA \& Isao WAKANO

New mathematical approach to the energy release rate in crack extension

MHF2006-17 Toru KOMATSU

Arithmetic of the splitting field of Alexander polynomial 